\title{
Mupirocin Resistance in Staphylococcus aureus Isolated from Nasal Swabs of ICU and OT Staff - A Study from A Tertiary Care Hospital
}

\author{
Sapna Chauhan ${ }^{1 *}$ (D) , Surender ${ }^{2}$ (D) and Tony J Rappai ${ }^{3}$ (D) \\ ${ }^{1}$ Department of Microbiology, Muzaffarnagar Medical College, Muzaffarnagar - 251 001, Uttar Pradesh, India. \\ ${ }^{2}$ Department of Anaesthesia, Muzaffarnagar Medical College, Muzafffarnagar - 251 001, Uttar Pradesh, India. \\ ${ }^{3}$ Department of Neurosurgery, Command Hospital, Lucknow 226002, India.
}

\begin{abstract}
Staphylococcus aureus is one of the common causes of Healthcare-associated infection. Staphylococcus colonizes the anterior nares of the nose and tends to disseminate and secondarily colonize several other body sites including the skin and the gut. Colonized hospital personnel may be an important factor in dissemination. Staphylococcus aureus to patients and vice-versa. Mupirocin is an excellent topical anti-staphylococcal antimicrobial agent used for eradicating nasal carriage. Resistance to Mupirocin is a threat for future use of this drug in eliminating nasal carriage of Staphylococcus aureus. Thus, this study was conducted to determine the rate of Mupirocin resistance among Staphylococcus aureus isolated from nasal swabs of Health care workers (HCWs) of Operation Theatres (OTs) and Intensive Care Units (ICUs). A single nasal swab was collected from both the anterior nares of participating health care workers of ICU and OT once at the end of their shift. Antibiotic susceptibility testing of Staphylococcus aureus to various antibiotics was done by Kirby-Bauer disk diffusion method using CLSI guidelines. High and low-level Mupirocin resistance was determined. Among 282 nasal swabs collected, Staphylococcus aureus was isolated in 62 samples. Of Staphylococcus aureus 19 came out to be Methicillin-resistant (MRSA) and the remaining 43 Methicillin sensitive (MSSA). Mupirocin resistance was seen in 3 MRSA strains and 1 MSSA strain. Thus, overall 4/62 (6.5\%) strains were MupR strains. Mupirocin is the most effective antibiotic used against colonization of Staphylococcus aureus in anterior nares. Resistance to this antibiotic is thus an alarm as well as a matter of great concern. Necessary steps, policies and guidelines need to be framed to stop the spread of this resistance.
\end{abstract}

Keywords: Mupirocin, Staphylococcus aureus, MRSA,MSSA, nasal swabs

*Correspondence: drsapna_chauhan@yahoo.com

(Received: March 26, 2021; accepted: September 01, 2021)

Citation: Chauhan S, Surender, Rappai TJ. Mupirocin Resistance in Staphylococcus aureus Isolated from Nasal Swabs of ICU and OT Staff- A Study from A Tertiary Care Hospital. J Pure Appl Microbiol. 2021;15(4):2059-2064. doi: 10.22207/JPAM.15.4.28

(C) The Author(s) 2021. Open Access. This article is distributed under the terms of the Creative Commons Attribution 4.0 International License which permits unrestricted use, sharing, distribution, and reproduction in any medium, provided you give appropriate credit to the original author(s) and the source, provide a link to the Creative Commons license, and indicate if changes were made. 


\section{INTRODUCTION}

Nosocomial or Health care associated infections (HAls) have become a potential threat to the world. Staphylococcus aureus especially the one that is Methicillin resistant is one of the leading cause of HAls. Staphylococcus colonizes various places and mucous membranes of the human body, $30 \%$ of the population is colonized worldwide especially in the anterior nares. ${ }^{1}$ Colonization can be transient - ranging from hours to days- or persistent over many months and years. Colonized hospital personnel may be an important factor in the dissemination from health care workers to patients and vice-versa, especially by HCWs working in OTs and ICUs, which are "hot zones" for the spread of drug resistance.

Mupirocin also known as pseudomonic acid $\mathrm{A}$ or by its tradename Bactroban is derived from Pseudomonas fluorescens and is an excellent topical anti-staphylococcal agent used for eradicating nasal carriage. ${ }^{2}$ It has been used to control outbreaks due to MRSA. Resistance to the drug was reported within 2 years of its introduction in the year 1987. ${ }^{3}$

Resistance to Mupirocin is of two types: Low level or intermediate resistance (MupL or Mupl) and high level resistance (MupH). The plasmid mediated Mup A and Mup B gene are responsible for high level resistance while low level resistance is associated with point mutations. ${ }^{4}$ These plasmids also carry resistance determinants to macrolides, gentamicin, tetracycline and trimethoprim. Hence resistance to Mupirocin would also select increased resistance to these antimicrobial agents. ${ }^{5}$

Mupirocin resistance is detected in laboratories using $5 \mu \mathrm{g}$ and $200 \mu \mathrm{g}$ discs. Resistance to $5 \mu \mathrm{g}$ disc but sensitive to $200 \mu \mathrm{g}$ is considered low level resistance while resistance to both $5 \mu \mathrm{g}$ and $200 \mu \mathrm{g}$ is considered high level resistance. While for low level resistance a increased dose of Mupirocin will work in vivo, a high level resistance would indicate treatment failure to Mupirocin. For these high-level drug resistant strains alternatives are only chlorhexidine, neomycin and novel agents like retapamulin., ${ }^{2,6}$

An increasing resistance to Mupirocin would indicate a major treatment loss against Methicillin resistant Staphylococcus aureus. Hence this study was undertaken so that HCWs can be screened for nasal carriage of Staphylococcus aureus, their resistance pattern could be known and they could be treated effectively and antibiotic policies could be made so as to prevent further spread of drug resistance.

\section{MATERIAL AND METHODS}

The study was a hospital based prospective study. Institutional ethical clearance was taken prior to the start of the study. Consent both informed and written was also obtained from all HCWs working in ICUs and OTs of Muzaffarnagar medical college \& hospital (a 750 bedded teaching hospital in Uttar Pradesh) for participating in the study.

A single nasal swab was used to take a mucosal sample from both anterior nares of participating HCWs, and transported to the Microbiology laboratory without delay. HCWs having upper respiratory tract infection and those with history of intake of antibiotics in previous three months were excluded from the study. While most of the doctors consented to give the sample, nursing staff was reluctant and did not consent.

Nasal swabs were inoculated onto blood agar and mannitol salt agar and incubated at $37^{\circ} \mathrm{C}$. Identification of Staphylococcus aureus was done on the basis of Gram staining, colony morphology and Biochemical tests like Coagulase, DNAase and Phosphatase test.

Antibiotic sensitivity was done using Kirby Bauer disk diffusion method. The zone diameters were interpreted as per Clinical and Laboratory Standards Institute (CLSI) guidelines. ${ }^{6}$ All the antibiotics were obtained from HiMedia Labs, India. The various antibiotics for which sensitivity was done were: Ampicillin, Amoxycillin-clavulanic acid, Ciprofloxacin, Clindamycin, Erythromycin,

Table 1. Distribution of Health care workers screened for Nasal carriage of Staphylococcus aureus

\begin{tabular}{lc}
\hline Category & $\begin{array}{c}\text { Number participating/total } \\
\text { number on staff of ICU or OT }\end{array}$ \\
\hline Doctors & $128 / 143$ \\
Nurses & $96 / 210$ \\
Technicians & $20 / 30$ \\
House keeping & $38 / 40$ \\
\hline
\end{tabular}

Those who did not consent or were having any respiratory illness or were on antibiotics were excluded from the study. 
Table 2. Distribution of Staphylococcus aureus isolated from various Health care workers

\begin{tabular}{lcc}
\hline Category & $\begin{array}{c}\text { Number } \\
\text { sampled }\end{array}$ & $\begin{array}{c}\text { Staphylococcus aureus } \\
\text { isolated from nose }\end{array}$ \\
\hline Doctors ICU/OT & 128 & $29(23 \%)$ \\
Nurses ICU/OT & 96 & $26(27 \%)$ \\
Technicians & 20 & $3(15 \%)$ \\
House keeping & 38 & $4(11 \%)$ \\
\hline
\end{tabular}

Table 3. Distribution of MRSA and MSSA amongst Staphylococcus aureus isolated

\begin{tabular}{lcc}
\hline Category & $\begin{array}{c}\text { Staphylococcus } \\
\text { aureus Isolated }\end{array}$ & MRSA \\
\hline Doctors & 29 & $12(41 \%)$ \\
Nurses & 26 & $6(23 \%)$ \\
Technicians & 3 & $1(33 \%)$ \\
House keeping & 4 & 0 \\
\hline
\end{tabular}

Cotrimoxazole, Tetracycline, Amikacin, Vancomycin and Linezolid. Methicillin resistance was detected using Cefoxitin disc as per CLSI guidelines. ${ }^{7}$

Mupirocin resistance was detected using $5 \mu \mathrm{g}$ and $200 \mu \mathrm{g}$ discs obtained from HiMedia labs, India. Staphylococcus aureus ATCC 25923 was taken as Quality control strain.

\section{RESULTS}

In our study nasal swabs were taken from 282 HCWs. The distribution of these health care workers is given in Table 1.

Out of these $282 \mathrm{HCWs}$ nasal swabs from 62 showed growth of Staphylococcus aureus. Maximum number of Staphylococcus aureus was isolated from Doctors, being 29 in number (Table 2).

Of these 62 Staphylococcus aureus isolated from nasal swabs 19 were Methicillin resistant (MRSA) and 43 were Methicillin sensitive (MSSA). The distribution of MRSA and MSSA is given in Table 3. While 12 MRSA were isolated from Doctors none of the house keeping member had even a single MRSA isolated.

Amongst these 62 Staphylococcus aureus isolated 1 showed Low level Mupirocin resistance while 3 showed High level Mupirocin resistance. The distribution of Mupirocin resistance both High level and Low level is mentioned in Table 4.

All of the Methicillin resistant Staphylococcus aureus were sensitive to Vancomycin and Linezolid while none was sensitive to Ampicillin, Amoxycillin-Clavulanic acid and Ciprofloxacin. Mupirocin resistant Staphylococcus aureus also showed same pattern (Table 5).

\section{DISCUSSION}

All the HCWs must be screened for being MRSA carrier especially those working in critical areas. These HCWs can be a potential source of infection for the patients. Detecting Mupirocin resistance in these MRSA strains becomes more important as Mupirocin is the most effective drug option used for eliminating nasal carriage of MRSA and resistance to this drug limits us to very few therapeutic options.

In our study we screened $282 \mathrm{HCW}$ working in either the ICU of Operating Theatre of a 750 bedded teaching hospital in India. Staphylococcus aureus was isolated from 62 of these HCWs. However the method used for collection and transport was not optimal as no enrichment was done. The carriage rate of Staphylococcus aureus in our study was $22 \%$. This finding is in accordance to studies by Rutvi $V$ et al. and Rongpharpi SR et al. ${ }^{8,9}$ An unusually high carriage rate was observed in a study by

Table 4. Distribution of Mupirocin high (MuH) and Mupirocin low level(MuL) resistance amongst Staphylococcus aureus isolated

\begin{tabular}{|c|c|c|c|c|c|}
\hline \multirow[t]{2}{*}{ Category } & \multirow{2}{*}{$\begin{array}{l}\text { Staphylococcus } \\
\text { aureus Isolated }\end{array}$} & \multicolumn{2}{|c|}{ MRSA(n=19) } & \multicolumn{2}{|c|}{$\operatorname{MSSA}(n=43)$} \\
\hline & & MuL & $\mathrm{MuH}$ & MuL & MuH \\
\hline Doctors & 29 & 0 & 2 & 1 & 0 \\
\hline Nurses & 26 & 0 & 1 & 0 & 0 \\
\hline Technicians & 3 & 0 & 0 & 0 & 0 \\
\hline House keeping & 4 & 0 & 0 & 0 & 0 \\
\hline
\end{tabular}


Table 5. Antibiotic Susceptibility profile of Methicillin and Mupirocin resistant Strains

\begin{tabular}{lcccc}
\hline Antibiotics & $\begin{array}{c}\text { Number of MRSA } \\
\text { strains }(\mathrm{n}=19)\end{array}$ & $\begin{array}{c}\text { Percentage } \\
\text { resistant }\end{array}$ & $\begin{array}{c}\text { Number of MupR } \\
\text { strains ( }=4)\end{array}$ & $\begin{array}{c}\text { Percentage } \\
\text { resistant }\end{array}$ \\
\hline Ampicillin & 19 & 100 & 4 & 100 \\
Amoxycillin-clavulanic & 19 & 100 & 4 & 100 \\
acid & & & & \\
Ciprofloxacin & 19 & 100 & 4 & 100 \\
Clindamycin & 12 & 63 & 3 & 75 \\
Erythromycin & 12 & 63 & 3 & 75 \\
Cotrimoxazole & 6 & 32 & 3 & 75 \\
Tetracycline & 3 & 16 & 3 & 75 \\
Amikacin & 11 & 58 & 4 & 0 \\
Vancomycin & 0 & 0 & 0 & 0 \\
Linezolid & 0 & 0 & 0 & \\
\hline
\end{tabular}

Kulshrestha $\mathrm{N}$ et al., the carriage in their study was 95.3\%. ${ }^{10}$

In the present study $19 \mathrm{HCW}$ were harboring MRSA in their nares. The carriage rate of MRSA comes out to be $6.7 \%$. This is similar to studies done by Rutvi $V$ et al. and Saleem M et al. ${ }^{8,11}$ Agarwal $L$ et al. have reported a higher carriage rate of MRSA in samples from doctors and sweepers while carriage rate in nurses was similar to our study. They reported carriage rate of MRSA as $13 \%, 6.7 \%$ and $14.2 \%$ in samples from doctors, nurses and sweepers, respectively. ${ }^{12}$ In a study done in Nigeria by Edem EN et al. $93 \%$ of HCWs were nasal carriers of Staphylococcus aureus and $20 \%$ of these were MRSA. ${ }^{13}$ The differences in nasal carriage rates of Staphylococcus aureus and MRSA may be contributed to differences in Infection control practices followed across the globe, but also in differences in detection methodology.

The nursing staff of the hospital was mostly reluctant to give the sample as many feared that they may be sent to leave or may be transferred from the OT, so even after counseling them many OT staff did not consent to give their sample. Hence the nasal carriage of nursing staff may not be true representation of the nasal carriage of Staphylococcus aureus. This is thus major limitation of the study.

Mupirocin resistance was also evaluated in Staphylococcus aureus isolated from HCWs. Overall mupirocin resistance was observed in 4/62 (6.5\%) Staphylococcus aureus isolates, they were isolated from three doctors and from one nurse.
This is in accordance to Indian study by Kumar $D$ et al. who reported mupirocin resistance in $13 \%$ of Staphylococcus aureus isolates. ${ }^{14}$ The nurse and one doctor was working in ICU while two doctors were from OT. All these HCWs were long term staff members of the hospital and they did not give any history of use of Mupirocin. In a study done by Elanithi K et al. none of Staphylococcus aureus was mupirocin resistant. ${ }^{15}$ International studies like one from France have reported none of Staphylococcus aureus to be Mupirocin resistant, similar to a study from Argentina where they have reported a low resistance of $3.1 \%{ }^{16,17}$

All of these Methicillinresistant strains were resistant to Ampicillin, Amoxycillin-clavulanic acid and Ciprofloxacin. Higher degree of resistance was seen for Clindamycin, Erythromycin and Amikacin being $63.12 \%, 63.12 \%$ and $57.89 \%$, respectively. All the methicillin resistant strains were sensitive to vancomycin and Linezolid. We also concluded that strains of Methicillin resistant were likely to besusceptible to tetracycline and cotrimoxazole, which is similar to study done by O'Neill et al. ${ }^{18}$

In the present study MupH was seen in only 3 and MupL in 1 of Staphylococcus aureus isolates. Gadepelli et al. have reported MupHamdMupL in $10(5 \%)$ and $2(1 \%)$ Staphylococcus aureus strains respectively. ${ }^{19}$ Oommen et al. have also reported MupH in only 1 (2.08\%) Staphylococcus aureus isolates, none MupL strain was found in their study. ${ }^{20}$

For nasal carriers of MRSA with MupH, Mupirocinnasal ointment does not clear the 
organism. MupH strains should be treated with chlorhexidine, neomycin or novelagents like retapamulin. ${ }^{6}$ However, MupL strains can be cleared by using higher mupirocin concentrations $(2000 \mathrm{~g} / \mathrm{ml})$. Such HCWs are usually advised to take baths with chlorhexidine scrubs and topical $2 \%$ mupirocin ointment application for 7 days, as well as change of duty or absence from duties till two culture negative reports are documented.

\section{CONCLUSION}

Mupirocin is the most effective antibiotic used against colonization of Staphylococcus aureus in anterior nares. Resistance to this antibiotic is thus an alarm as well as matter of great concern. Necessary steps, policies and guidelines need to be framed to stop spread of this resistance. A strategy of regularly screening HCWs would help prevent the spread of Methicillin and of Mupirocin resistant Staphylococcus aureus among HCWs as well as through them to patients and thereby to the community.

\section{ACKNOWLEDGMENTS}

None.

\section{CONFLICT OF INTEREST}

The authors declare that there is no conflict of interest.

\section{AUTHORS' CONTRIBUTION}

All authors listed have made a substantial, direct and intellectual contribution to the work, and approved it for publication.

\section{FUNDING}

None.

\section{DATA AVAILABILITY}

All datasets generated or analysed during this study are included in the manuscript.

\section{ETHICS STATEMENT}

The study was approved by Institutional Ethics Committee Muzaffarnagar Medical College, UP, India (MMC/IEC/2021/237)

\section{REFERENCES}

1. Kluytmans J, van Belkum A, Verbrugh H. Nasal carriage of Staphylococcus aureus: epidemiology, underlying mechanisms, and associated risks. Clin Microbiol Rev. 1997;10(3):505-520. doi: 10.1128/CMR.10.3.505

2. Wattal C, Oberoi JK. Mupirocin resistant staphylococcus aureus nasal colonization among healthcare workers. Indian J Crit Care Med. 2014;18(11):709-710. doi: 10.4103/0972-5229.144009

3. Rahman M, Noble WC, Cookson B, D Baird, J Coia. Mupirocin-resistant Staphylococcus aureus. Lancet. 1987;330(8555):387-388. doi: 10.1016/S01406736(87)92398-1

4. Seah C, Alexander DC, Louie L, et al. MupB, a new high-level mupirocin resistance mechanism in Staphylococcus aureus. Antimicrob Agents Chemother. 2012;56(4):1916-1920. doi: 10.1128/AAC.05325-11

5. Patel JB, Gorwitz RJ, Jernigan JA. Mupirocin resistance. Clin Infect Dis. 2009;49(6):935-941. doi: 10.1086/605495

6. Patel $A B$, Jennifer $L$, Fulmer $Y$, Richard $C$, Ratner AJ, Shopsin B. Retapamulin Activity Against Pediatric Strains of Mupirocin-resistant Methicillinresistant Staphylococcus aureus. Paediatr Infect Dis J. 2021;40(7):637-638. doi: 10.1097/ INF. 0000000000003123

7. Clinical and laboratory standards institute, performance standards for antimicrobial susceptibility testing; twenty fourth informational supplement.CLSI document M100-S23.Wayne, PA; 2018.

8. Rutvi V, Sangeeta DP, Sima KB, Piyush AP. Nasal Carriage Rate of Methicillin Resistant Staphylococcus aureus (MRSA) among Civil Hospital Health care workers. Intl J Med Public Health. 2016;6(4):180-183. doi: 10.5530/ ijmedph.2016.4.7

9. Rongpharpi SR, Hazarika NK, Kalita H. The prevalnace of Nasal carriage of Staphylococcus aureus among health care workers at a tertiary care hospital in Assam with special reference to MRSA. J Clin Diag Res. 2013;7(2):257-260. doi: 10.7860/ JCDR/2013/4320.2741

10. Kulshrestha N, Ghatak T, Gupta P, Singh M, Agarwal J. Surveillance of health-care workers for nasal carriage to detect multidrug-resistant Staphylococcus spp. in a tertiary care center: An observational study. Med J DY Patil Vidyapeeth. 2019;12(1):39-43. doi: 10.4103/ mjdrdypu.mjdrdypu_74_18

11. Saleem M, Ahmad I, Alenazi. Incidence of clinical isolates and its antimicrobial resistance pattern among the nasal and hand swabs of operation theatre staff in tertiary and secondary care hospitals. Biomedical Research. 2018;29(18):3408-3414. doi: 10.4066/ biomedicalresearch.29-17-1503

12. Agarwal L, Singh AK, Agarwal A, Agarwal A. Methicillin and mupirocin resistance in nasal colonizers coagulase negative Staphylococcus among health care workers. Med J DY Patil Univ. 2016;9(4):479-483. doi: 10.4103/0975-2870.186070

13. Edem EN, Onwuezobe IA, Ochang EA, Etok CA, James IS. Antibiogram of bacterial isolates from the anterior nares and hands of health care workers in university of Uyo teaching hospital (UUTH) Uyo, Akwalbom state, Nigeria. J Bacteriol Parasitol. 2013;4(2):168. doi: 10.4172/2155-9597.1000168

14. Kumar D, Bisht D, Faujdar SS. Incidence of Mupirocin 
Resistance in Staphylococcus aureus Isolated from Rural Population: A New Emerging Challenge. Int J Curr Res Rev. 2020;12 (22):82-85. doi: 10.31782/ IJCRR.2020.12225

15. Elanithi K, Kavitha E, Easow JM. Screening for Nasal Carriage of Mupirocin Resistant Staphylococcus aureus among health care workers in a tertiary care hospital. Indian J Microbiol Res. 2018;5(2):240-243. doi: 10.18231/2394-5478.2018.0050

16. Trouillet-Assant S, Flammier S, Sapin A, et al. Mupirocin Resistance in Isolates of Staphylococcus spp. from Nasal Swabs in a Tertiary Hospital in France. J Clin Microbiol. 2015;53(8):2713-2715. doi: 10.1128/ JCM.00274-15

17. Boncompain CA, Suarez CA, Morbidon HR. Staphylococcus aureus nasal carriage in health care workers: First report from a major public hospital in Argentina. Rev Argent Microbiol. 2017;49(2):125-231. doi: 10.1016/j.ram.2016.12.007

18. O'Neill AJ, McLaws F, Kahlmeter G, Henriksen AS, Chopra I. Genetic basis of resistance to fusidic acid in Staphylococci. Antimicrob Agents Chemother. 2007;51(5):1737-1740. doi: 10.1128/AAC.01542-06

19. Gadepalli R, Dhawan B, Mohanty S, et al. Mupirocin resistance in Staphylococcus aureus in an Indian hospital. Diagn Microbiol Infect Dis. 2007;58(1):125127. doi: 10.1016/j.diagmicrobio.2006.10.012

20. Oommen SK, Appalaraju B, Jinsha K. Mupirocin resistance in clinical isolates of staphylococci in a tertiary care centre in south India. Indian J Med Microbiol. 2010;28(4):372-375. doi: 10.4103/02550857.71825 